\title{
Circulating fibrocytes as prognostic biomarkers of autoimmune interstitial lung disease
}

\author{
John Odackal ${ }^{1}$, Victor Yu ${ }^{1}$, Diana Gomez-Manjerres ${ }^{2}$, Joshua J. Field ${ }^{3}$, \\ Marie D. Burdick ${ }^{1,2}$ and Borna Mehrad (10 ${ }^{2}$
}

Affiliations: ${ }^{1}$ Dept of Medicine, University of Virginia School of Medicine, Charlottesville, VA, USA. ${ }^{2}$ Division of Pulmonary, Critical Care, and Sleep Medicine, University of Florida, Gainesville, FL, USA. ${ }^{3}$ Medical Sciences Institute and Blood Research Institute, Blood Center of Wisconsin, Milwaukee, WI, USA.

Correspondence: Borna Mehrad, Box 100225, University of Florida, Gainesville, FL 32610-0225, USA. E-mail: Bailea.Gnanndamedicine.ufl.edu

\section{ABSTRACT}

Background: Autoimmunity is a common cause of pulmonary fibrosis and can present either as a manifestation of an established connective tissue disease or as the recently described entity of interstitial pneumonia with autoimmune features. The rate of progression and responsiveness to immunosuppression in these illnesses are difficult to predict. Circulating fibrocytes are bone marrow-derived progenitor cells that home to injured tissues and contribute to lung fibrogenesis. We sought to test the hypothesis that the blood fibrocyte concentration predicts outcome and treatment responsiveness in autoimmune interstitial lung diseases.

Methods: We compared the concentration of circulating fibrocytes in 50 subjects with autoimmune interstitial lung disease and 26 matched healthy controls and assessed the relationship between serial peripheral blood fibrocyte concentrations and clinical outcomes over a median of 6.25 years.

Results: As compared to controls, subjects with autoimmune interstitial lung disease had higher circulating concentrations of total fibrocytes, the subset of activated fibrocytes, and fibrocytes with activation of PI3K/AKT/mTOR, transforming growth factor- $\beta$ (TGF- $\beta$ ) receptor and interleukin (IL)- $4 / \mathrm{IL}$ 13 receptor signalling pathways. Over the follow-up period, there were episodes of marked elevation in the concentration of circulating fibrocytes in subjects with autoimmune interstitial lung disease but not controls. Initiation of immunosuppressive therapy was associated with a decline in the concentration of circulating fibrocytes. For each $100000 \mathrm{cells} \cdot \mathrm{mL}^{-1}$ increase in peak concentration of circulating fibrocytes, we found a $5 \%$ increase in odds of death or lung function decline.

Conclusion: In patients with autoimmune interstitial lung disease, circulating fibrocytes may represent a biomarker of outcome and treatment response.

@ERSpublications

Autoimmune diseases are common causes of pulmonary fibrosis. The blood concentration of fibrocytes, cells involved in formation of scar tissue, predicts outcomes and response to immunosuppression in these patients. https://bit.ly/35bel62

Cite this article as: Odackal J, Yu V, Gomez-Manjerres D, et al. Circulating fibrocytes as prognostic biomarkers of autoimmune interstitial lung disease. ERJ Open Res 2020; 6: 00481-2020 [https:// doi.org/10.1183/23120541.00481-2020].

This article has supplementary material available from openres.ersjournals.com

Availability of data and materials: The datasets used during the current study are available from the corresponding author on reasonable request.

Received: 8 July 2020 | Accepted after revision: 29 Aug 2020

Copyright $\odot$ ERS 2020. This article is open access and distributed under the terms of the Creative Commons Attribution Non-Commercial Licence 4.0. 


\section{Introduction}

Autoimmunity is among the most common causes of interstitial lung disease (ILD). Approximately $40 \%$ of patients with a diagnosis of rheumatoid arthritis, systemic sclerosis, autoimmune myopathies, mixed connective tissue disease and primary Sjögren syndrome have ILD [1], and conversely, many of the patients who present with an undiagnosed ILD have evidence of a previously undiagnosed connective tissue disease [2]. In addition, the recently defined entity of interstitial pneumonia with autoimmune features describes patients with evidence of autoimmunity who do not meet the diagnostic criteria for a connective tissue disease [3] and represents another important subset of autoimmune ILD. Together, these illnesses comprise a large proportion of patients seen by ILD specialists and present unique challenges relating to diagnosis, therapy and prognosis.

The rate of progression among autoimmune ILDs is notoriously heterogeneous and difficult to predict, with many patients displaying mild lung disease or a slowly progressive course, but a subset presenting with rapid progression to respiratory failure and death [4]. The rate of response to immunosuppressive therapies can also be unpredictable [5]. Clinical measures of ILD - including pulmonary function tests (PFTs), computed tomography (CT) imaging pattern and histology - provide aggregate evidence of past episodes of inflammatory and fibrotic damage to the lungs but have limited value in predicting future events [6]. As such, identification of biomarkers to predict prognosis and response to therapy in these illnesses would constitute an important clinical advance and is an area of research priority [7].

Fibrocytes are bone marrow-derived circulating progenitor cells that can home to sites of injury and contribute to scar formation [8]. In mouse models of fibrotic lung disease, fibrocytes rapidly exit the bone marrow to enter the bloodstream after tissue injury and home to the lungs; blocking this migration attenuates lung fibrosis [9-11]. In human ILD, elevated circulating fibrocyte concentration is associated with subsequent poor outcome in idiopathic pulmonary fibrosis and in a monogenic form of ILD [12, 13]. Elevation of circulating fibrocytes has previously been observed in fibrotic lung diseases associated with rheumatoid arthritis and scleroderma [14-17], but, to our knowledge, the value of longitudinal measurements of fibrocyte concentration in predicting the outcome of autoimmune ILD has not been reported to date.

In the current study, we hypothesised that the blood fibrocyte concentration predicts outcome and treatment responsiveness in autoimmune ILD. To test this hypothesis, we performed a cohort study in which we correlated serial peripheral fibrocyte counts with PFTs, response to therapy and survival over time.

\section{Materials and methods}

Subjects and study visits

We recruited healthy subjects and subjects with autoimmune ILD between October 2010 and July 2015. At enrolment, subjects with ILD underwent a thorough history and physical examination, serological workup for autoimmune disease and high-resolution chest CT. Diagnoses of autoimmune diseases were adjudicated by two study team members ( $\mathrm{OO}$ and $\mathrm{BM}$ ) in accordance with published guidelines for rheumatoid arthritis [18], scleroderma [19], mixed connective tissue disease [20], myositis-related ILD [21] and interstitial pneumonia with autoimmune features [3]. Usual interstitial pneumonia (UIP) was identified based on histology or when lung biopsies had not been performed, "definite UIP pattern" on high-resolution chest CT [22]. Initiation of immunosuppressive therapy was defined as initiation of azathioprine ( $\geqslant 50 \mathrm{mg}$ daily) or mycophenolate mofetil ( $\geqslant 1000 \mathrm{mg}$ daily) after $\geqslant 3$ months off these medications, or increase in prednisone by $\geqslant 10 \mathrm{mg}$ daily after $\geqslant 3$ months on a stable dose of immunosuppressive medications. At each visit, PFTs were performed per established guidelines [23, 24], and venous blood was collected. PFT variables were expressed as per cent of predicted values of the National Health and Nutrition Examination Survey (NHANES)-III database. PFT decline was defined as either a reduction of forced vital capacity (FVC) by $\geqslant 10 \%$ of predicted value or diffusing capacity of the lung for carbon monoxide $\left(D_{\mathrm{LCO}}\right)$ by $\geqslant 15 \%$ predicted value. Study follow-up ended in January 2018, when death or censure were assigned by reviewing electronic medical records and publicly available death records.

\section{Sample processing and flow cytometry}

Blood samples were collected in heparinised tubes and immediately placed on ice, then refrigerated overnight before processing for fibrocytes quantification by flow cytometry without ex vivo manipulations such as culture or enrichment, as previously described [13, 25-27]. See the online supplementary material (supplementary appendix S1) for detailed methods and gating strategy.

\section{Statistical analyses}

Data were analysed in SAS (version 9.4 for Windows; SAS Institute, Cary, NC, USA) or Prism (version 8 for Mac; GraphPad, San Diego, CA, USA). Descriptive data were summarised as median and interquartile 
range (IQR). Differences in demographics of subjects with stable disease and those with death or PFT decline were assessed using Wilcoxon rank-sum or Fisher's exact tests. Fisher's exact test was also used to assess the association between PFT decline and death. Log-rank test was used to access the association between UIP pattern and death. In cross-sectional comparisons, groups were compared using the Wilcoxon rank-sum test. Univariable and multivariable logistic regression was used to predict combined outcome of death or decline in functional status. For survival outcome classification, Youden's J-index was calculated to define threshold of maximum total fibrocyte count. A Chi-squared test was performed for relative risk of death or functional decline above and below threshold of maximum total fibrocyte count. A Wilcoxon signed-rank test was performed to assess the effect of immunosuppression on paired fibrocyte counts. Results were considered significant where the two-sided p-value $<0.05$.

\section{Results}

We recruited 26 healthy controls and 50 subjects with autoimmune ILD (table 1). Among subjects with lung disease, 16 died and five experienced a decline in PFT during the 6.25-year study period (figure 1a), but death was not significantly associated with PFT decline (figure 1b). Among the subjects without PFT decline, the FVC increased by a median of $4 \%$ (IQR 0 to $12 \%$ ) and $D_{\text {LCO }}$ did not change (IQR -9 to $+10 \%)$ over the study period. The subjects who died or with PFT decline did not differ from subjects with stable disease in baseline characteristics (supplementary table S1). UIP pattern was associated with death at 40 months of enrolment (log-rank, $\mathrm{p}=0.01)$.

In a cross-sectional comparison, we found the median concentration of circulating fibrocytes from the initial sample obtained from subjects with autoimmune ILD to be 2.5 -fold higher in subjects with ILD as compared to healthy controls (figure 2a). The majority of the circulating fibrocytes expressed the chemokine receptor CXCR4, with smaller subsets expressing CCR2, CCR5 and CCR7; all these subsets were significantly higher in subjects with autoimmune ILD. Fibrocytes expressing the haematopoietic stem cell marker CD34, while significantly higher in subjects with ILD, constituted a small subset of total circulating fibrocytes (supplementary figure S1).

To compare the activation state of fibrocytes between groups, we quantified the absolute concentration of circulating fibrocytes that express the myofibroblast differentiation marker, $\alpha$-smooth muscle actin, and found this subset to also be expanded in patients with autoimmune ILD (figure $2 \mathrm{~b}$ ). To assess key fibrotic signalling pathways within circulating fibrocytes, we quantified the fibrocytes that contained the phosphorylated forms of transcription factors involved in transforming growth factor- $\beta$ (TGF- $\beta$ ) receptor signalling (Smad-2 and -3), the PI3K/AKT/mTOR pathway (AKT-1 and P70S6K), and interleukin (IL)-4

\section{TABLE 1 Summary of demographic and clinical data of the study subjects}

\begin{tabular}{|c|c|c|c|}
\hline & Healthy controls & ILD subjects & p-value \\
\hline Subjects $\mathrm{n}$ & 26 & 50 & \\
\hline Age years & $55(48-62)$ & $60(50-69)$ & 0.07 \\
\hline Male sex & $11(41)$ & $21(42)$ & 0.97 \\
\hline \multicolumn{4}{|l|}{ Race } \\
\hline Caucasian & $14(54)$ & $37(74)$ & \\
\hline African American & $7(27)$ & $13(26)$ & 0.53 \\
\hline \multicolumn{4}{|l|}{ Diagnosis } \\
\hline IPAF & & $18(36)$ & \\
\hline Rheumatoid arthritis & & $5(10)$ & \\
\hline MCTD & & $3(6)$ & \\
\hline Myositis-related & & $13(26)$ & \\
\hline Scleroderma & & $11(22)$ & \\
\hline \multicolumn{4}{|c|}{ Pulmonary function tests } \\
\hline FVC $\%$ predicted & & $61.5(51-78)$ & \\
\hline$D_{\text {Lco }} \%$ predicted & & $42.5(26-55)$ & \\
\hline UIP pattern & & $11(22)$ & \\
\hline \multicolumn{4}{|c|}{ Supplemental oxygen use } \\
\hline Subjects $n(\%)$ & & $15(20)$ & \\
\hline Flow rate $L \cdot \min ^{-1}$ & & $2-3$ & \\
\hline $\begin{array}{l}\text { Data are presented as } \\
\text { disease; IPAF: interstiti } \\
\text { FVC: forced vital capaci }\end{array}$ & $\begin{array}{l}\text { tile rangel or } \mathrm{n}(\% \\
\text { h autoimmune fea } \\
\text { capacity for carbo }\end{array}$ & $\begin{array}{l}\text { herwise stated. } \\
\text { D: mixed conne } \\
\text {; UIP: usual int }\end{array}$ & $\begin{array}{l}\text { itial lung } \\
\text { disease; } \\
\text { umonia. }\end{array}$ \\
\hline
\end{tabular}



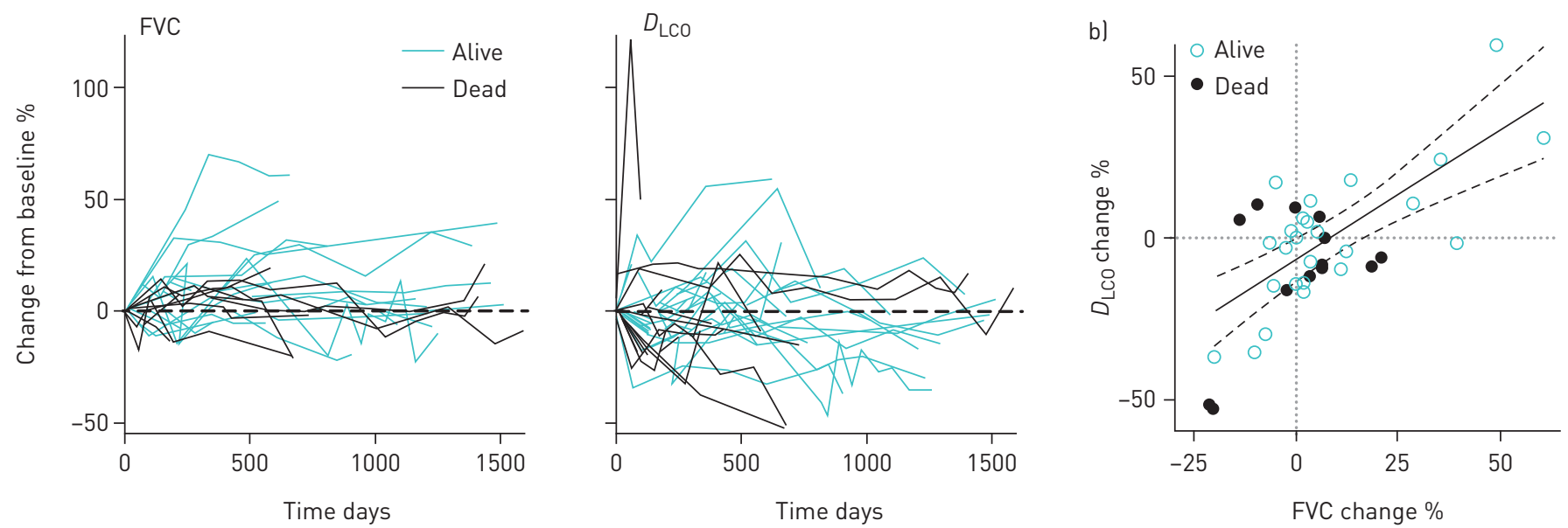

FIGURE 1 Trends in pulmonary function tests (PFTs). a and b) Trends in forced vital capacity (FVC) and diffusing capacity of the lung for carbon monoxide $\left(D_{\mathrm{LCO}}\right)$ over time for interstitial lung disease (ILD) subjects. Each line represents one subject. Dashed line represents no change. C) Change between first and last PFT over the study period. Solid and dashed lines represent correlation and $95 \%$ confidence interval; dotted lines represent no change.

and IL-13 receptor signalling (STAT6). We found the subset of fibrocytes with activation in these signalling pathways to be significantly higher in subjects with autoimmune ILD as compared to healthy controls (figure 2c-f). The most highly expressed activation markers expressed by fibrocytes from ILD
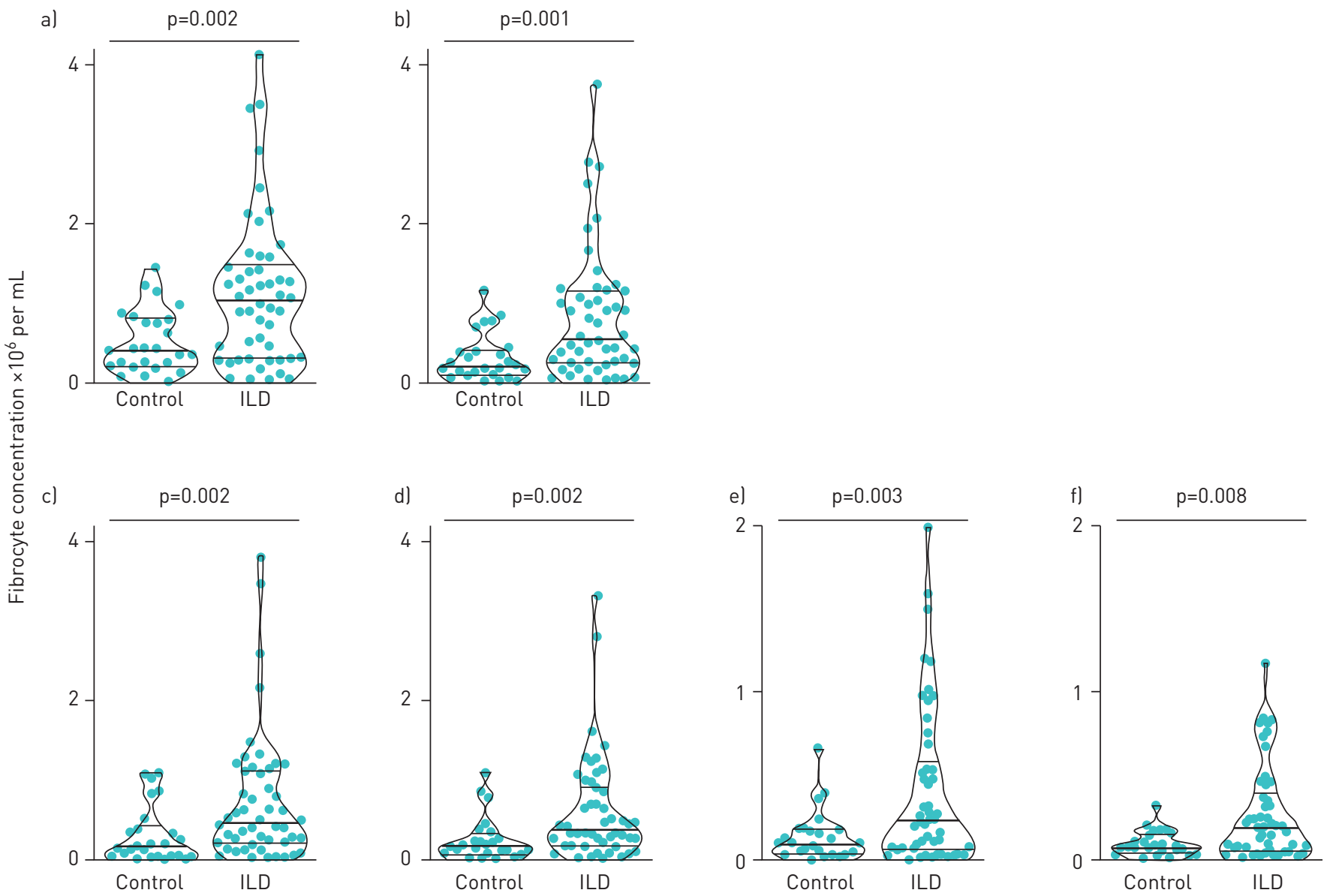

FIGURE 2 Cross-sectional comparison of circulating fibrocyte concentration in subjects with autoimmune interstitial lung disease (ILD) and healthy controls. a) Total circulating fibrocytes (CD45 ${ }^{+}$Col $1^{+}$cells); b) activated fibrocytes (CD45 ${ }^{+}$Col $1^{+}$aSMA ${ }^{+}$cells); c-f) fibrocytes staining for phosphorylated Smad-2/3, AKT-1, P70SK6 and STAT6, respectively. Each dot represents one subject; bold horizontal lines in the violin plots show the median and light horizontal lines represent the 25th and 75th percentiles. 
patients were $\alpha$-smooth muscle actin (expressed by a median of $77 \%$ of fibrocytes), phosphorylated Smad-2/3 (69\%) and phosphorylated AKT (56\%), with a minority of fibrocytes expressing phosphorylated P70S6K and STAT-6 (28\% and 19\%, respectively). We next assessed the longitudinal change in absolute circulating fibrocyte concentrations in 40 subjects with autoimmune ILD and seven healthy control subjects. Serial samples exhibited episodes of marked elevation of fibrocyte concentration in subjects with autoimmune ILD but not in healthy control subjects (figure 3). During the study period, 13 subjects with autoimmune ILD had fibrocyte concentrations measured both before and after initiation or escalation of immunosuppression. In this group, the median interval between pre- and post-treatment fibrocyte analysis was 88 days (IQR 54-121). Immunosuppressive therapy was associated with a $43 \%$ decline in median absolute concentration of circulating fibrocytes and a $41 \%$ decline in the activated subset of fibrocytes (figure 4).

In univariable analysis of the entire cohort, the peak fibrocyte concentration of subjects with autoimmune ILD predicted death or PFT decline: each increase in peak fibrocyte concentration of $10^{5}$ cells $\mathrm{mL}^{-1}$ was associated with a $5 \%$ increase in the odds of death or PFT decline (table 2). UIP pattern and gender-age-physiology score (a predictive index based on sex, age, predicted $D_{\text {LCO }}$ and FVC) have each been shown to predict outcomes in autoimmune lung disease [28-30]; we therefore performed multivariable logistic regression models to include these known predictors. Inclusion of these variables in the model did not change the relationship between peak fibrocyte concentration and outcome (table 2). We identified a threshold peak fibrocyte concentration of $2.6 \times 10^{6}$ fibrocytes $\cdot \mathrm{mL}^{-1}$ to be associated with doubling of the relative risk of death or PFT decline over the study period (95\% CI 1.08-4.1; $\mathrm{p}=0.036$ ).

\section{Discussion}

Clinicians detect worsening of ILD only when it is severe enough to distort lung anatomy macroscopically, as detected by high-resolution chest CT, or to impair whole-organ physiology, as detected by PFT. There are two limitations to these assessments: first, CT and PFT are insensitive to early, and perhaps reversible, changes in lung inflammation and scarring; and second, these modalities measure the aggregate of damage to the lung by past insults, which may not correlate to future deteriorations of lung disease and death from respiratory insufficiency. Cellular and biochemical biomarkers of the underlying pathophysiology have the potential to overcome these limitations and provide information that is complementary to such traditional clinical measurements.

A number of biomarkers have been investigated for their utility in making a diagnosis and determining the trajectory of autoimmune ILD: the pattern of autoantibodies can categorise patients according to the risk of developing ILD; proteins derived from respiratory epithelium, including the cell surface glycoprotein Mucin-1 (also known as Krebs von den Lugen-6) and surfactant proteins, correlate with the degree of lung injury; and plasma cytokines and acute phase proteins have been used as surrogates of active inflammation [7, 31]. Few studies, however, have focused on biomarkers of fibrogenesis as predictors of outcome in autoimmune lung diseases, and none, to our knowledge, have assessed the predictive value of longitudinal measurements of such biomarkers over time.
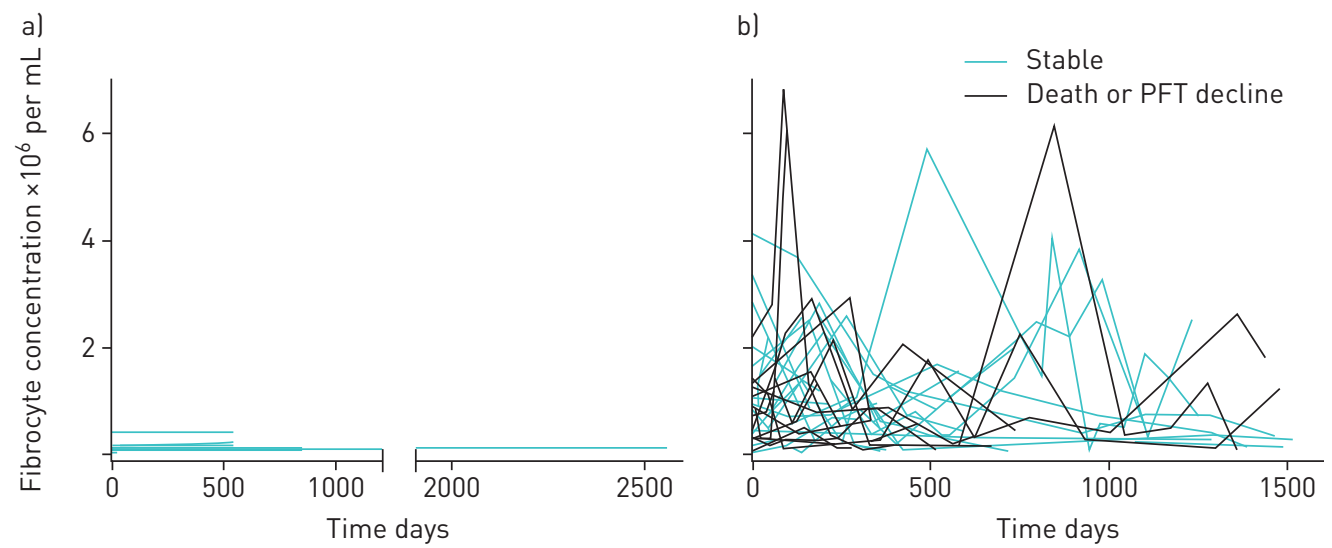

FIGURE 3 Change in circulating fibrocyte concentration over study period among a) healthy controls and b. subjects with autoimmune interstitial lung disease. Each line represents one subject. PFT: pulmonary function test. 
a)

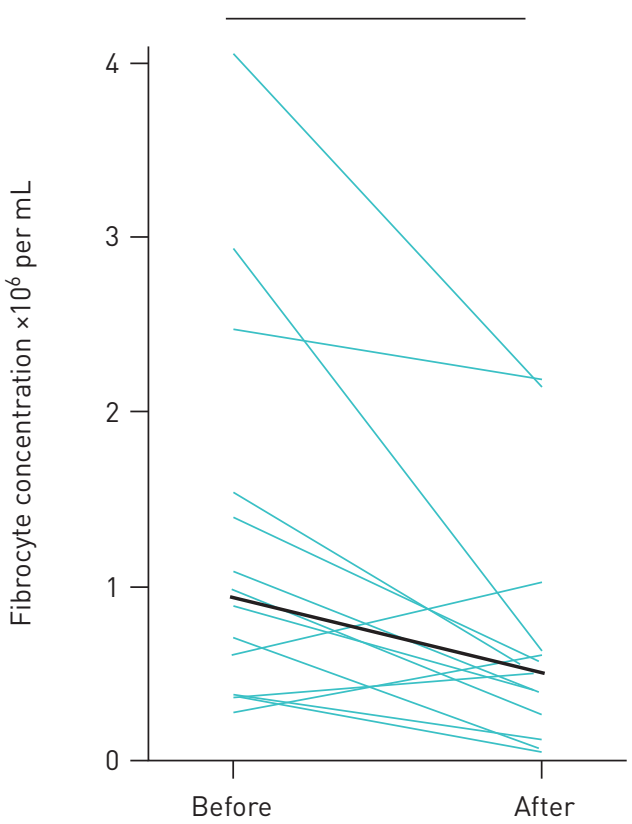

b)

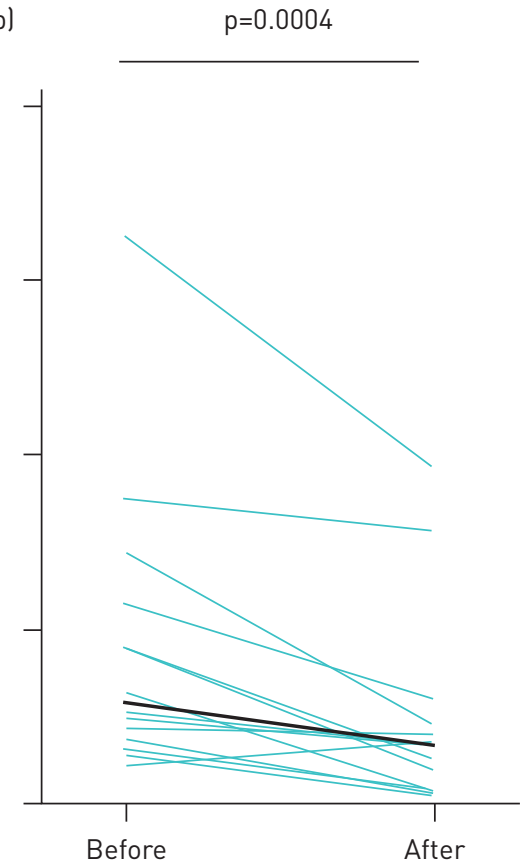

FIGURE 4 Effect of immunosuppressive therapy on circulating fibrocyte concentration. a) Total circulating fibrocytes $\left(\mathrm{CD}_{4} 5^{+} \mathrm{Col} 1^{+}\right.$cells) and b) activated fibrocytes (CD45 ${ }^{+} \mathrm{Col} 1^{+} \mathrm{aSMA}{ }^{+}$cells) are shown. Each green line represents one sample; black lines represent median values.

Fibrocytes are released from the bone marrow in response to diverse forms of tissue injury. The mechanism by which fibrocytes contribute to physiological wound healing and pathological scarring of injured organs is controversial in the literature, with some publications supporting differentiation into myofibroblasts and others providing evidence for a paracrine role in promoting fibrosis [11, 32-34]. Regardless of mechanism, the blood concentration of these cells may serve as an easily measurable marker, not of the extent of organ injury, but the fibrogenic response to that injury. This hypothesis was supported by a study in idiopathic pulmonary fibrosis, in which the proportion of fibrocytes in peripheral blood buffy coat leukocytes at a single time point correlated with concurrent acute exacerbations and was predictive of subsequent death [12]. We next performed a longitudinal study in Hermansky-Pudlak syndrome, a rare autosomal recessive disease, that afforded us the opportunity to describe the behaviour of circulating fibrocytes over time in a relatively homogeneous population of patients with ILD [13]. Unexpectedly, many patients in this population exhibited episodic and marked elevations of the absolute concentration of circulating fibrocytes over time, the magnitude of which was predictive of death from respiratory failure, but interestingly did not correlate with PFT decline [13]. We hypothesised that, at least in patients with this form of ILD, episodes of elevation in fibrocytes in the bloodstream reflect abrupt episodes of lung injury, which cumulatively result in lung function deterioration and respiratory failure.

In the current study, we sought to extend the prior findings to the more common, but far more heterogeneous, category of ILD attributable to autoimmune diseases. Prior work has shown fibrocytes to

\section{TABLE 2 Logistic regression to predict death or functional decline}

\begin{tabular}{|c|c|c|c|}
\hline \multirow[t]{2}{*}{ Parameter } & \multicolumn{3}{|c|}{ OR estimate $(95 \% \mathrm{CI})$} \\
\hline & Univariate model & Multivariate model 1 & Multivariate model 2 \\
\hline Subjects n & 50 & 50 & 49 \\
\hline $\begin{array}{l}\text { Peak fibrocyte concentration, per } \\
100000 \text { increase }\end{array}$ & $1.044(1.002-1.088)$ & $1.050(1.007-1.096)$ & 1.050 (1.005-1.097) \\
\hline UIP pattern & & $3.292(0.783-13.84)$ & \\
\hline GAP stage II or III & & & 2.324 (0.649-8.319) \\
\hline
\end{tabular}


be elevated in the bloodstream of patients with ILD due to rheumatoid arthritis and scleroderma [14, 16]. Fibrocytes are detectable in the lungs of patients with scleroderma-associated ILD [17] and have been mechanistically linked to ILD in this population $[15,35,36]$. The present work adds to this literature by showing that, similar to patients with Hermansky-Pudlak syndrome, subjects with autoimmune ILD develop episodic marked elevations in circulating fibrocytes that were predictive of death or decline in pulmonary function. Importantly, the predictive value of fibrocytes was cumulative and continuous, conferring a $5 \%$ increased risk per $10^{5}$ increase in fibrocyte concentration, and was independent of the gender-age-physiology score and UIP pattern, each of which have previously been linked to worse outcomes in autoimmune ILD [28, 29, 37, 38]. The threshold value of fibrocyte concentration identified in this study is similar to the values we identified in patients with Hermansky-Pudlak syndrome, chronic sickle cell lung disease and post-acute respiratory distress syndrome (ARDS) pulmonary fibrosis [13, 27, 39].

The current study also extends the prior literature by assessing several intracellular signalling events relevant to fibrogenesis in circulating fibrocytes. By detecting epitopes that result from specific amino-acid phosphorylations of transcription factors, flow cytometry is a powerful tool for identifying the signalling landscape in rare cell types with minimal ex vivo manipulation [40]. We detected the majority of circulating fibrocytes to contain phosphorylated Smad-2 and -3 , evidence for TGF- $\beta$ receptor signalling in these cells [41]. In addition, fibrocytes in autoimmune ILD, but not control subjects, expressed phosphorylated forms of AKT1 and P70SK6, indicating activation of the PI3K/AKT/mTOR pathway. The latter is implicated in lung fibrogenesis and, in particular, in fibrocyte activation [11, 42]. Lastly, we identified a subset of fibrocytes that express phosphorylated STAT- 6 in the context of autoimmune ILD. Although not previously described in fibrocytes, STAT-6 is an important mechanism in fibrosis and is relevant to fibroblast activation $[43,44]$, and prior work has documented the response of fibrocytes to IL-4 and IL-13 [45, 46].

We recognise several limitations in our study. The sample size was relatively small, reducing the study power and increasingly the likelihood of false-negative findings. This study was performed at a single tertiary care centre, raising questions about the generalizability of its findings. Although fibrocytes have been predictive of outcome in other ILDs, the current study does include a second validation cohort of patients with autoimmune ILD. By studying autoimmune ILD as a single entity, the study was not powered to detect differences in predictive utility of fibrocytes in individual diseases or in response to specific immunosuppressive drugs. Finally, the data on the response of fibrocytes to immunosuppressive therapy is based on a small number of patients and should be viewed as hypothesis-generating rather than definitive.

The findings of this study suggest several avenues for future research. First, the utility of fibrocytes as biomarkers of prognosis and response to therapy in patients with autoimmune ILD should be compared to other biomarkers. In this regard, combinations of serum biomarkers have been associated with better detection of rheumatoid arthritis-associated ILD than any single biomarker [31]; a similar approach may inform biomarkers of prognosis. Second, we report a fall in circulating fibrocytes in the subset of subjects who were started on immunosuppressive therapy during our study period. This observation suggests the hypothesis that fibrocytes may be useful as a biomarker of response to immunosuppression, for example identifying patients who have not responded to therapy, and conversely, guiding de-escalation of immunosuppression in patients with quiescent disease.

Finally, based on the results of the current study and prior studies of fibrocytes in other fibrotic lung diseases, we propose a general model wherein progression of diverse forms of ILD is the consequence of repeated fibrogenic responses to mostly subclinical episodes of alveolar epithelial injury. We propose that these responses include the release of fibrocytes from bone marrow into the bloodstream, which can be detected on scheduled screening blood tests, for example during routine clinic visits. This model is consistent with the reduction in blood fibrocyte concentration after immunosuppressive therapy which, we posit, suppresses epithelial injury and thus the fibrogenic response to that injury. This hypothesis can be tested in longitudinal studies of different subtypes of ILD.

Author contributions: Conceptualisation: B. Mehrad. Data curation: J. Odackal, V. Yu, M.D. Burdick, B. Mehrad. Data analysis: J. Odackal, M.D. Burdick, B. Mehrad. Funding acquisition: B. Mehrad. Investigation: D. Gomez-Manjerres, J.J. Field, M.D. Burdick, B. Mehrad. Methodology: M.D. Burdick, B. Mehrad. Administration: M.D. Burdick, B. Mehrad. Resources: J.J. Field, B. Mehrad. Supervision: M.D. Burdick, B. Mehrad. Visualisation: J. Odackal, M.D. Burdick, B. Mehrad. Writing the original draft: J. Odackal. Critical revision of the manuscript: all authors.

Ethics approval and consent to participate: We obtained informed consent from the subjects in accordance with the Declaration of Helsinki, according to an institutionally approved protocol (University of Virginia IRB-HSR 15299). 
Conflict of interest: J. Odackal has nothing to disclose. V. Yu has nothing to disclose. D. Gomez-Manjerres has nothing to disclose. J.J. Field has nothing to disclose. M.D. Burdick has nothing to disclose. B. Mehrad holds a patent on the clinical use of fibrocyte measurements in fibrotic interstitial lung diseases.

Support statement: This work was funded by NIH grants HL098329, AI135128 and EB024501. Funding information for this article has been deposited with the Crossref Funder Registry.

\section{References}

1 Mira-Avendano I, Abril A, Burger CD, et al. Interstitial lung disease and other pulmonary manifestations in connective tissue diseases. Mayo Clin Proc 2019; 94: 309-325.

2 Cotton CV, Spencer LG, New RP, et al. The utility of comprehensive autoantibody testing to differentiate connective tissue disease associated and idiopathic interstitial lung disease subgroup cases. Rheumatology (Oxford) 2017; 56: 1264-1271

3 Fischer A, Antoniou KM, Brown KK, et al. An official European Respiratory Society/American Thoracic Society research statement: interstitial pneumonia with autoimmune features. Eur Respir J 2015; 46: 976-987.

4 Antoniou KM, Margaritopoulos G, Economidou F, et al. Pivotal clinical dilemmas in collagen vascular diseases associated with interstitial lung involvement. Eur Respir J 2009; 33: 882-896.

5 Adegunsoye A, Strek ME. Therapeutic approach to adult fibrotic lung diseases. Chest 2016; 150: 1371-1386.

6 Hansell DM, Goldin JG, King TE, et al. CT staging and monitoring of fibrotic interstitial lung diseases in clinical practice and treatment trials: a position paper from the Fleischner Society. Lancet Respir Med 2015; 3: 483-496.

7 Bonella F, Costabel U. Biomarkers in connective tissue disease-associated interstitial lung disease. Semin Respir Crit Care Med 2014; 35: 181-200.

8 Mehrad B, Strieter RM. Fibrocytes and the pathogenesis of diffuse parenchymal lung disease. Fibrogenesis Tissue Repair 2012; 5: Suppl. 1, S22.

9 Moore BB, Murray L, Das A, et al. The role of CCL12 in the recruitment of fibrocytes and lung fibrosis. Am J Respir Cell Mol Biol 2006; 35: 175-181.

10 Field JJ, Burdick MD, DeBaun MR, et al. The role of fibrocytes in sickle cell lung disease. PLoS One 2012; 7: e33702.

11 Mehrad B, Burdick MD, Strieter RM. Fibrocyte CXCR4 regulation as a therapeutic target in pulmonary fibrosis Int J Biochem Cell Biol 2009; 41: 1708-1718.

12 Moeller A, Gilpin SE, Ask K, et al. Circulating fibrocytes are an indicator of poor prognosis in idiopathic pulmonary fibrosis. Am J Respir Crit Care Med 2009; 179: 588-594.

13 Trimble A, Gochuico BR, Markello TC, et al. Circulating fibrocytes as biomarker of prognosis in Hermansky-Pudlak syndrome. Am J Respir Crit Care Med 2014; 190: 1395-1401.

14 Just SA, Lindegaard H, Hejbøl EK, et al. Fibrocyte measurement in peripheral blood correlates with number of cultured mature fibrocytes in vitro and is a potential biomarker for interstitial lung disease in Rheumatoid Arthritis. Respir Res 2017; 18: 141

15 Galligan CL, Keystone EC, Fish EN. Fibrocyte and T cell interactions promote disease pathogenesis in rheumatoid arthritis. J Autoimmun 2016; 69: 38-50.

16 Mathai SK, Gulati M, Peng X, et al. Circulating monocytes from systemic sclerosis patients with interstitial lung disease show an enhanced profibrotic phenotype. Lab Invest 2010; 90: 812-823.

17 Tourkina E, Bonner M, Oates J, et al. Altered monocyte and fibrocyte phenotype and function in scleroderma interstitial lung disease: reversal by caveolin-1 scaffolding domain peptide. Fibrogenesis Tissue Repair 2011 ; 4 : 15.

18 Aletaha D, Neogi T, Silman AJ, et al. 2010 Rheumatoid arthritis classification criteria: an American College of Rheumatology/European League Against Rheumatism collaborative initiative. Arthritis Rheum 2010; 62: 2569-2581.

19 van den Hoogen F, Khanna D, Fransen J, et al. 2013 classification criteria for systemic sclerosis: an American College of Rheumatology/European League against Rheumatism collaborative initiative. Arthritis Rheum 2013; 65 2737-2747.

20 Alarcón-Segovia D, Cardiel MH. Comparison between 3 diagnostic criteria for mixed connective tissue disease Study of 593 patients. J Rheumatol 1989; 16: 328-334.

21 Lega JC, Reynaud Q, Belot A, et al. Idiopathic inflammatory myopathies and the lung. Eur Respir Rev 2015; 24: $216-238$.

22 Lynch DA, Sverzellati N, Travis WD, et al. Diagnostic criteria for idiopathic pulmonary fibrosis: a Fleischner Society White Paper. Lancet Respir Med 2018; 6: 138-153.

23 Miller MR, Hankinson J, Brusasco V, et al. Standardisation of spirometry. Eur Respir J 2005; 26: 319-338.

24 Macintyre N, Crapo RO, Viegi G, et al. Standardisation of the single-breath determination of carbon monoxide uptake in the lung. Eur Respir J 2005; 26: 720-735.

25 Keeley EC, Schutt RC, Marinescu MA, et al. Circulating fibrocytes as predictors of adverse events in unstable angina. Transl Res 2016; 172: 73-83.

26 Shipe R, Burdick MD, Strieter BA, et al. Number, activation, and differentiation of circulating fibrocytes correlate with asthma severity. J Allergy Clin Immunol 2016; 137: 750-757.

27 Lin CM, Alrbiaan A, Odackal J, et al. Circulating fibrocytes traffic to the lung in murine acute lung injury and predict outcomes in human acute respiratory distress syndrome: a pilot study. Mol Med 2020; $26: 52$.

28 Kim HC, Ji W, Kim MY, et al. Interstitial pneumonia related to undifferentiated connective tissue disease: pathologic pattern and prognosis. Chest 2015; 147: 165-172.

29 Ryerson CJ, Vittinghoff E, Ley B, et al. Predicting survival across chronic interstitial lung disease: the ILD-GAP model. Chest 2014; 145: 723-728.

30 Walsh SL, Sverzellati N, Devaraj A, et al. Connective tissue disease related fibrotic lung disease: high resolution computed tomographic and pulmonary function indices as prognostic determinants. Thorax 2014; 69: 216-222.

31 Doyle TJ, Patel AS, Hatabu H, et al. Detection of rheumatoid arthritis-interstitial lung disease is enhanced by serum biomarkers. Am J Respir Crit Care Med 2015; 191: 1403-1412. 
Lassance L, Marino GK, Medeiros CS, et al. Fibrocyte migration, differentiation and apoptosis during the corneal wound healing response to injury. Exp Eye Res 2018; 170: 177-187.

33 Ashley SL, Wilke CA, Kim KK, et al. Periostin regulates fibrocyte function to promote myofibroblast differentiation and lung fibrosis. Mucosal Immunol 2017; 10: 341-351.

34 Madala SK, Edukulla R, Schmidt S, et al. Bone marrow-derived stromal cells are invasive and hyperproliferative and alter transforming growth factor- $\alpha$-induced pulmonary fibrosis. Am J Respir Cell Mol Biol 2014; 50: 777-786.

35 Sun $\mathrm{H}$, Zhu $\mathrm{Y}$, Pan $\mathrm{H}$, et al. Netrin-1 regulates fibrocyte accumulation in the decellularized fibrotic sclerodermatous lung microenvironment and in bleomycin-induced pulmonary fibrosis. Arthritis Rheumatol 2016; 68: 1251-1261

36 Reese C, Perry B, Heywood J, et al. Caveolin-1 deficiency may predispose African Americans to systemic sclerosis-related interstitial lung disease. Arthritis Rheumatol 2014; 66: 1909-1919.

37 Morisset J, Vittinghoff E, Lee BY, et al. The performance of the GAP model in patients with rheumatoid arthritis associated interstitial lung disease. Respir Med 2017; 127: 51-56.

38 Marie I, Hatron PY, Dominique S, et al. Short-term and long-term outcomes of interstitial lung disease in polymyositis and dermatomyositis: a series of 107 patients. Arthritis Rheum 2011; 63: 3439-3447.

39 Mehrad B, Burdick MD, Wandersee NJ, et al. Circulating fibrocytes as biomarkers of impaired lung function in adults with sickle cell disease. Blood Adv 2017; 1: 2217-2224.

40 Hedley DW, Chow S, Shankey TV. Cytometry of intracellular signaling: from laboratory bench to clinical application. Methods Cell Biol 2011; 103: 203-220.

41 Derynck R, Budi EH. Specificity, versatility, and control of TGF- $\beta$ family signaling. Sci Signal 2019; 12: eaav5183.

42 Lawrence J, Nho R. The role of the mammalian target of rapamycin (mTOR) in pulmonary fibrosis. Int J Mol Sci 2018; 19: 778

43 Gieseck RL, Wilson MS, Wynn TA. Type 2 immunity in tissue repair and fibrosis. Nat Rev Immunol 2018; 18: 62-76.

44 Doucet C, Brouty-Boyé D, Pottin-Clémenceau C, et al. Interleukin (IL) 4 and IL-13 act on human lung fibroblasts. Implication in asthma. J Clin Invest 1998; 101: 2129-2139.

45 Bellini A, Marini MA, Bianchetti L, et al. Interleukin (IL)-4, IL-13, and IL-17A differentially affect the profibrotic and proinflammatory functions of fibrocytes from asthmatic patients. Mucosal Immunol 2012; 5: 140-149.

46 Maharjan AS, Pilling D, Gomer RH. High and low molecular weight hyaluronic acid differentially regulate human fibrocyte differentiation. PLoS One 2011; 6: e26078. 